\title{
An efficient way to screen inhibitors of energy- coupling factor (ECF) transporters in a bacterial uptake assay
}

Spyridon Bousis, $\ddagger^{a, b, c}$ Steffen Winkler, $\neq^{a}$ Jörg Haupenthal, ${ }^{a}$ Francesco Fulco, ${ }^{a}$ Eleonora

Diamanti, ${ }^{a, d}$ and Anna K. H. Hirsch *a,b,c,d

a. Department for Drug Design and Optimization, Helmholtz Institute for Pharmaceutical Research (HIPS) - Helmholtz Centre for Infection Research (HZI), Campus Building E 8.1, D66123, Saarbrücken, Germany.

b. Stratingh Institute for Chemistry, University of Groningen, Nijenborgh 7, NL-9747 AG Groningen, the Netherlands.

c. Department of Pharmacy, Saarland University, Campus Building E8.1, 66123 Saarbrücken, Germany.

d. Helmholtz International Lab for Anti-Infectives Campus E8.1, 66123 Saarbrücken (Germany)

\section{KEYWORDS}

Energy-coupling factor transporters, antimicrobials, B-type vitamins, bacterial uptake assay, screening

\section{ABSTRACT}

Herein, we report a novel whole-cell screening assay using Lactobacillus casei as model microorganism to identify inhibitors of energy-coupling factor (ECF) transporters. This promising and underexplored target may have important pharmacological potential through modulation of vitamin homeostasis in bacteria and, importantly, it is absent in humans. The assay represents an alternative, cost-effective and fast solution to demonstrate the direct involvement of these membrane transporters in a native biological environment rather than using a low-throughput in 
vitro assay employing reconstituted proteins in a membrane bilayer system. Based on this new whole-cell screening approach, we demonstrated the optimization of a weak hit compound (2) into a small molecule (3) with improved in vitro and whole-cell activities. This study opens the possibility to quickly identify novel inhibitors of ECF transporters and optimize them based on structure-activity relationships.

\section{Introduction}

Energy-coupling factor (ECF) transporters are a class of transmembrane proteins that facilitate the uptake of micronutrients such as B-type vitamins and cations inside the cell. They belong to the superfamily of ATP-binding cassette (ABC) transporters and actively import several micronutrients by consumption of ATP. The architecture of the ECF transporters consists of a complex of four proteins: one transmembrane protein (T-component), two cytosolic ATPases (Acomponents) forming together the energy-coupling module (EcfAA'T), and a substrate-specific binding protein (S-component). ${ }^{1}$ Based on the genomic location of the S-components, they are classified into groups I and II (Figure 1). 


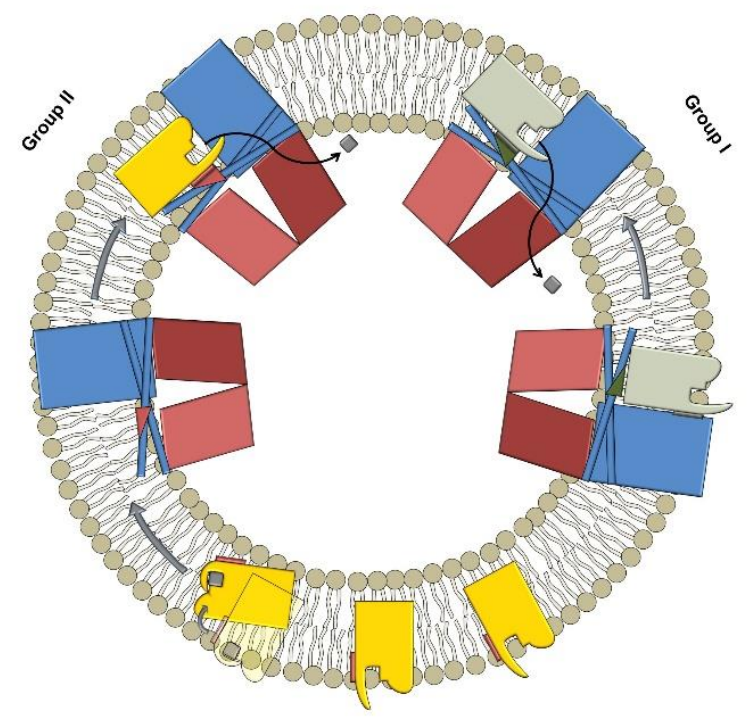

Figure 1. Schematic representation of group I and II ECF transporters. The heterodimeric ATPases (ECFA and ECFA') are represented in light and dark red color, while the T-component is shown in blue. The S-components belonging to the ECF-group I and II are colored in light green and yellow, respectively.

Specifically, in group I a "dedicated" EcfAA'T interacts with a single S-component and in this class, both EcfAA'T and S-component are encoded in the same operon. For instance, both CbiMNQO and NikMNQO belong to group I of ECF transporters that mediate cobalt and nickel ion uptake, respectively. ${ }^{2}$ Regarding group II, a single "shared” EcfAA'T interacts with various Scomponents, and is responsible for the import of various micronutrients. In this case, the module and the substrate-specific protein are not encoded in the same operon, but copies of different Scomponents are scattered across in the chromosome. ECF FolT and ECF ThiT are two examples of this subgroup (II) that transport folic acid and thiamine, respectively. ${ }^{3}$ The importance of the ECF transporters resides in their taxonomic distribution; they are present only in prokaryotes and, 
in contrast to other $\mathrm{ABC}$ transporters, they are absent from eukaryotes, making them promising targets for the treatment of bacterial infections. A large number of bacteria, including pathogens such as Enterococcaceae, Streptococcaceae and Clostridia utilize ECF transporters to maintain the homeostasis of several vitamins. ${ }^{4}$ For example, Streptococcus pneumoniae uses ECF transporters to salvage a variety of B-type vitamins such as pantothenate, riboflavin, niacin, pyridoxal and biotin. These pathogens cause a significant nosocomial and economic burden. ${ }^{5}$ In addition, some of them, such as vancomycin-resistant Enterococcus faecium and penicillin-non-susceptible S. pneumoniae, are classified by the World Health Organization as high and medium priority pathogens for the development of new antibiotics, respectively. ${ }^{6}$ Furthermore, ECF transporters are also present in non-pathogenic organisms such as in bacteria of the genus Lactobacillus. Specifically, Lactobacillus lactis and Lactobacillus delbrueckii have been extensively used as model microorganisms, providing valuable insights into the transport mechanism as well as the physical 3D conformation of the ECF transporters. ${ }^{1}$ Notably, L. lactis is one of the first organisms that was used to study the transport of folic acid into the bacterium mediated by an ECF transporter. ${ }^{7}$ Although ECF transporters have been recently reported as potential antibiotic targets, they remain underexplored from a medicinal point of view. In order to identify and evaluate novel inhibitors by cell-based screening the use of an assay that allows for a certain throughput is necessary. ECF transporters are transmembrane proteins, therefore their purification is challenging, while the stability of both the whole complex and the ECF module is trivial. Following our first publication, in which we used an in vitro transport-activity assay on purified ECF FolT2 reconstituted in proteoliposomes to identify hits 1 and $\mathbf{2}$ (Figure 2) ${ }^{8}$, we here present the establishment of a convenient functional, whole cell-based transport assay using $L$. casei in 96-well plates. 

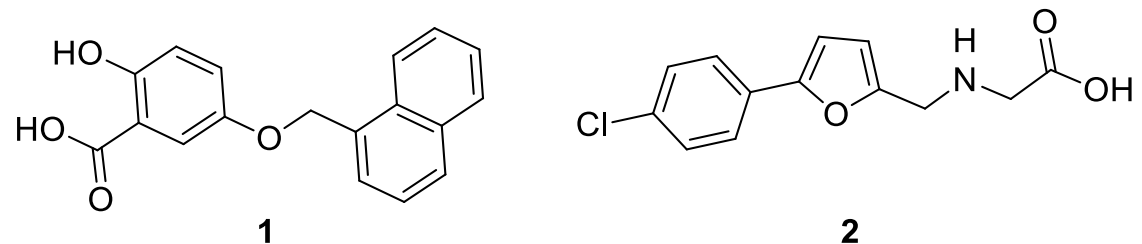

Figure 2. Chemical structures of the two identified inhibitors of the group II ECF-FolT2 transporter.

The new whole-cell uptake assay was used to further validate and develop hit $\mathbf{2}$ into a more potent compound through a structure-activity relationship (SAR) study improving its properties and antibacterial activity. This achievement was accomplished by two complementary approaches: biochemical evaluation of a commercial library of structurally related compounds of hit $\mathbf{2}$ and a classical SAR study.

\section{Results and Discussion}

Inspired by the way Henderson ${ }^{7}$ measured the transport of various vitamins mediated by ECF transporters, we developed a functional bacterial uptake assay to evaluate the ability of molecules to inhibit transport of vitamins by an ECF group-II transporter.

In our previous study, we showed that bacterial cells can be used efficiently to biologically evaluate compounds as potential inhibitors of an ECF transporter. ${ }^{8}$ Specifically, an Escherichia coli strain that expresses heterologous ECF FolT2 from L. delbrueckii was used demonstrate on target activity. In the present study, we selected a Gram-positive strain (L. casei) due to its ability to import several essential vitamins such as folic acid, riboflavin, thiamine and biotin from the environment. This strain encodes all the necessary genes for the expression of EcfAA'T as well as the S-components (FolT, RibU, ThiT and BoiY) that interact with and bind to the respective vitamins. $^{7}$ 
Although L. casei is not pathogenic, it constitutes an excellent choice as it is one of the best-studied model microorganisms for the transport of folic acid and other vitamins mediated by ECF transporters. Furthermore, it has no additional barriers (outer membrane) that the compounds need to overcome to enter the bacteria. Since L. casei is auxotrophic for folic acid, it was chosen over other strains that regulate the homeostasis of folate by both uptake and de novo biosynthesis. It is considered that the uptake would be higher in an auxotrophic strain. In an attempt to imitate the physiological conditions we developed a transport assay in L. casei instead of E. coli, as the ECF transporters are naturally present in Gram-positive but not in Gram-negative strains. A bacterial uptake assay has many advantages over an in vitro assay (Table 1).

Table 1. Advantages of a bacterial uptake assay over uptake into proteoliposomes. The bacterial uptake assay is performed in L. casei, whereas the uptake into proteoliposomes is based on the isolated ECF FolT2 transporter from L. delbrueckii.

\begin{tabular}{|l|l|}
\hline Bacterial uptake assay & Uptake into Proteoliposomes \\
\hline Medium to high throughput & Low throughput \\
\hline No protein purification & Requires protein purification \\
\hline Multiparameter optimization & Single-parameter optimization \\
\hline Monitor the uptake of several vitamins & Monitor the uptake of specific vitamins \\
\hline
\end{tabular}

Importantly, the speed of testing increased dramatically as this assay can be performed in 96-well format. The challenging step of purifying a transmembrane protein can be avoided, reducing the amount of work. In addition, the bacterial uptake assay is a multiparameter assay as the efficacy and the permeability across biological barriers are considered. Last but not least, the allosteric mode of inhibition can be addressed by changing the substrate (vitamin imported by different Scomponents), while in an in vitro setup, a second protein needs to be purified and reconstituted in liposomes. In consequence, inhibition of the uptake of different vitamins by the desired compounds 
are pointing to a targeted, allosteric inhibition of the energy-coupling module rather than inhibiting the S-component, leading to reduced uptake of all ECF transporter-mediated vitamins and a multifaceted disturbance of metabolic cellular activity and growth.

The development of the bacterial uptake assay required recurring iterative optimization of the assay conditions. Initially, we observed a strongly varying uptake rate in different cultures. Because cellular activity strongly varies during different growth phases, including enzyme expression levels, the availability of nutrients or viabilities, the assay was preceded with precultivation of $L$. casei reaching the beginning of the exponential growth phase $\left(\mathrm{OD}_{600}=0.5\right)$. Furthermore, possible interfering substances, such as residual vitamins in the medium, were removed prior to the assay by resuspending the cells in citrate buffer including $2 \%$ D-glucose. Glucose had been reported to enhance the uptake rate of folic acid in L. casei by increasing the ATP availability. ${ }^{9}$ This is reasonable, since it is the co-substrate in ATPases and the driving force of the ECF transporter. Besides, 96-well filter plates in combination with a vacuum manifold were used, which markedly accelerated washing steps compared to centrifuge-based separation techniques ensuring a rapid screening of test compounds.

To further investigate folic acid uptake in $L$. casei, Michaelis-Menten kinetics were analyzed reporting a $K_{M}$ of $49( \pm 4) \mathrm{nM}$ (Figure S9) that is similar to other published values. Both compounds $\mathbf{1}$ and $\mathbf{2}$ were designed to inhibit the EcfT domain allosterically - altering the maximal uptake rate, but not the $K_{M}$. Thus, the folic acid concentration could be adjusted to a level that induces uptake rates close to the maximum uptake rate (5-10 times higher than $K_{M}$ ), where the effects of allosteric inhibitors are supposed to be more visible and competitive inhibitors are identified as less active compounds. However, higher folate concentrations would have either reduced the fraction of radiolabeled folate or increased the total amount of radiolabeled folate. In 
turn, this resulted either in a lower signal and a lower accuracy of the assay or in markedly higher costs, so that the folate working concentration was set to $50 \mathrm{nM}$. Furthermore, the allosteric mode can be further investigated by using other vitamins, such as riboflavin, thiamine or biotin, as discussed above.

Finally, the $\mathrm{IC}_{50}$ of $\mathbf{1}$ was determined at $282 \pm 108 \mu \mathrm{M}$ for the uptake into proteoliposomes assay and it exhibited an inhibition of $40 \% @ 250 \mu \mathrm{M}$ the bacterial uptake assay showing a comparable potency between the two assays (Figure S10). Thus, hit 1 was chosen as positive control for all following assays. To ensure the compounds have time to enter the bacteria and steady state binding of the target, the compounds were mixed with the bacterial suspension and incubated prior of starting the transport reaction by folate addition. Impairments that may be caused by DMSO as an organic solvent for the test compounds, such as decreased viability or further unforeseen side effects, were not observed for DMSO working concentrations of up to $10 \%$, allowing the screening of more hydrophobic compounds (Figure S11).

Having established the transport assay using $\mathbf{1}$ as reference compound, firstly we measured the inhibition of the uptake of folic acid by 2 (Figure 3). Whereas 2 exhibits an $\mathrm{IC}_{50}$ value of $1.2 \mathrm{mM}$ in the in vitro assay in $L$. delbrueckii, it is inactive up to $1.2 \mathrm{mM}$ in the bacterial uptake assay $L$. casei. $^{8}$ We reasoned that the difference in activity may be ascribed either to permeability issues of the compound or to a difference in the amino acid sequence of the ECF transporter between $L$. delbrueckii and L. casei. Even if both assays rely on ECF transporters, differences both in the sequence and in the nature of the assay may cause variations in the activity. 


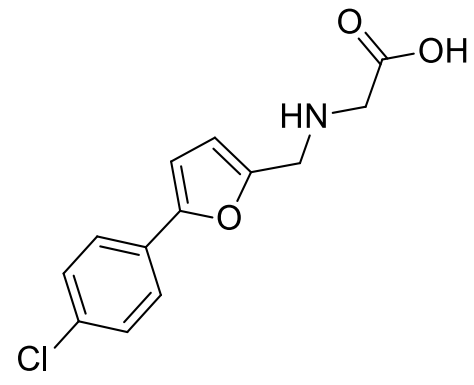

2

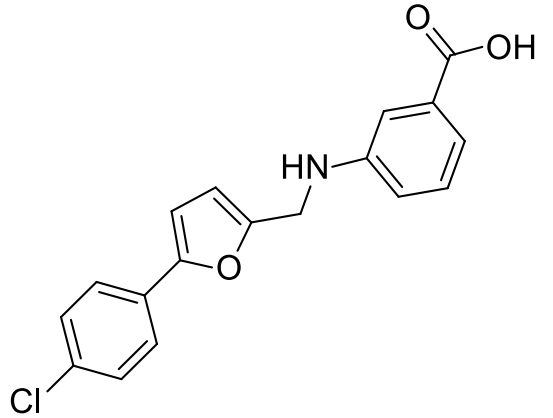

3

\begin{tabular}{|c|c|c|}
\hline Name & $\mathrm{IC}_{50}$ & Assay type of ECF-FolT \\
\hline $\mathbf{2}$ & $>1.2 \mathrm{mM}$ & Proteoliposome uptake \\
\hline $\mathbf{3}$ & $174 \mu \mathrm{M}$ & Bacterial uptake \\
\hline
\end{tabular}

Figure 3. Chemical evolution of $\mathbf{2}$ to $\mathbf{3}$ presented on this work.

In order to explore the chemical space around compound 2, we extended our screening to a small library of structurally related compounds, which were previously obtained through commercial sources or in-house chemical synthesis. Most of the compounds were tested at $500 \mu \mathrm{M}$, and less soluble ones were tested at their maximum soluble concentration. From the nineteen compounds (4-22) tested, seven $(\mathbf{4}, \mathbf{5}, \mathbf{1 0}, \mathbf{1 1}$ and $\mathbf{1 6}-\mathbf{1 8})$ showed more than $20 \%$ inhibition, while the others did not show any ability to influence the transportation of the ECF transporters (Figure S12).

In parallel, to address the permeability issue caused by the zwitterionic nature of $\mathbf{2}$, we rationally designed and synthesized molecules with broad modifications and devoid of zwitterionic nature. To do so, we substituted the secondary aliphatic amine with an aniline maintaining the carboxylic acid. Interestingly derivative $\mathbf{3}$, which bears the $\mathrm{COOH}$ group in $m$-position, is seven-fold more potent compared to the initial hit $\mathbf{2}$ (Table 2).

Table 2. SAR of the aniline derivatives. Biological evaluation of $\mathbf{3}$ and 19-22 for inhibition of the ECF FolT transporter-mediated folate import in the L. casei based uptake assay. 


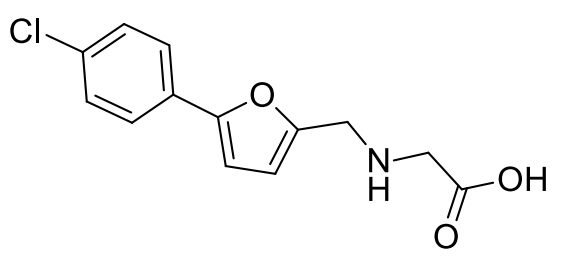

2

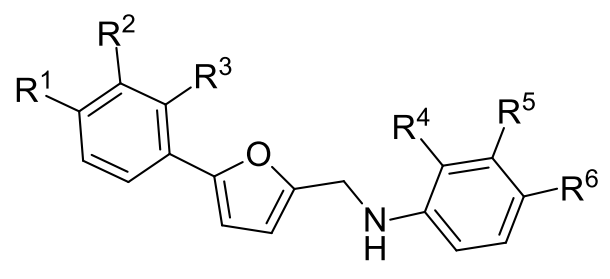

$3,19-22$

\begin{tabular}{|c|c|c|c|c|c|c|c|}
\hline Name & $\mathrm{R}^{1}$ & $\mathrm{R}^{2}$ & $\mathrm{R}^{3}$ & $\mathrm{R}^{4}$ & $\mathrm{R}^{5}$ & $\mathrm{R}^{6}$ & inhibition @ 200 $\mu \mathrm{M}$ \\
\hline $\mathbf{3}$ & $\mathrm{Cl}$ & $\mathrm{H}$ & $\mathrm{H}$ & $\mathrm{H}$ & $\mathrm{COOH}$ & $\mathrm{H}$ & $43 \%$ \\
& & & & & & & $\left(\mathrm{IC}_{50}=177 \mu \mathrm{M}\right)$ \\
\hline $\mathbf{2 3} *$ & $\mathrm{Cl}$ & $\mathrm{H}$ & $\mathrm{H}$ & $\mathrm{COOH}$ & $\mathrm{H}$ & $\mathrm{H}$ & $11 \% @ 50 \mu \mathrm{M}$ \\
\hline $\mathbf{2 4}$ & $\mathrm{Cl}$ & $\mathrm{H}$ & $\mathrm{H}$ & $\mathrm{H}$ & $\mathrm{H}$ & $\mathrm{COOH}$ & n.i @ 500 $\mu \mathrm{M}$ \\
\hline $\mathbf{2 5}$ & $\mathrm{H}$ & $\mathrm{Cl}$ & $\mathrm{H}$ & $\mathrm{H}$ & $\mathrm{COOH}$ & $\mathrm{H}$ & $41 \%$ \\
\hline $\mathbf{2 6}$ & $\mathrm{H}$ & $\mathrm{H}$ & $\mathrm{Cl}$ & $\mathrm{H}$ & $\mathrm{COOH}$ & $\mathrm{H}$ & $36 \%$ \\
\hline
\end{tabular}

*Not soluble above $50 \mu \mathrm{M}$. n.i. $=$ no inhibition (if inhibition $<10 \%$ ).

Then, we tested the sensitivity of the ortho- and para-position of the $\mathrm{COOH}$ group within the distal phenyl ring (23 and 24). While compound 23 turned out to be insoluble under the conditions of the assay, 24 showed no inhibition up to $500 \mu \mathrm{M}$. Next, we explored the left-hand ring that bears the chloride atom, and observed that changing the substitution pattern of the chloride from parato meta- to ortho- does not influence the cell-based activity (see $\mathbf{3}, \mathbf{2 5}$, and $\mathbf{2 6}$, respectively Table 2). This finding makes the left-hand ring a good place for potential fragment growing. In a third step, we designed molecules having modifications in the middle furan ring. Therefore, we shifted the substitution pattern from ${ }_{1} \mathrm{C}-4 \mathrm{C}$ to ${ }_{1} \mathrm{C}-3 \mathrm{C}(\mathbf{2 7})$, which surprisingly abolished the activity of the molecule (Table 3). 
Table 3. SAR of the derivatives that bear modifications at the middle ring. Biological evaluation of $\mathbf{3}$ and $\mathbf{2 7}$ for inhibition of the ECF FolT transporter-mediated folate import in the L. casei-based uptake assay.

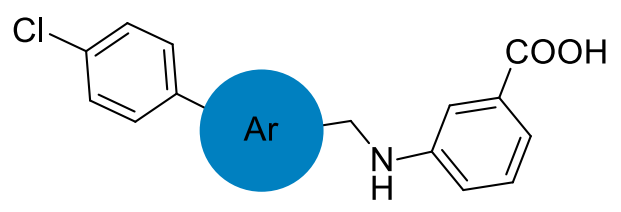

\begin{tabular}{|c|c|c|}
\hline Name & $\mathrm{Ar}$ & inhibition@200 $\mathrm{MM}$ \\
\hline 3 & & $43 \%$ \\
\hline 27 & & $8 \%$ \\
\hline
\end{tabular}

To examine the antimicrobial activity of $\mathbf{3}$ as the best compound in this study, a panel of Grampositive pathogens that rely on the ECF have been screened. Encouragingly, $\mathbf{3}$ showed a good antimicrobial activity against both S. aureus and S. pneumoniae strains (Table 4). ${ }^{4}$

Table 4. Antibacterial profile of 3 .

\begin{tabular}{|l|c|c|}
\hline Indicator strain & Auxotrophic & $\begin{array}{c}\text { MIC } \\
(\mu \mathrm{g} / \mathrm{mL})\end{array}$ \\
\hline Staphylococcus aureus str. Newman & no & 23 \\
\hline Streptococcus pneumoniae DSM-20566 & yes & 21 \\
\hline Streptococcus pneumoniae DSM-11865 $^{[\mathrm{a}]}$ & yes & 10 \\
\hline
\end{tabular}

The auxotrophic status indicates which of the strains is auxotrophic for at least one B-vitamin. [a] PRSP: penicillin-resistant Streptococcus pneumoniae. 


\section{CONCLUSIONS}

We established a working platform based on 96-well plates that allows for a quick and convenient identification of ECF transporter inhibitors. This protocol is based on a bacterial uptake assay using $L$. casei as model microorganism. The assay has the advantage of identifying compounds that inhibit ECF transporters in a whole-cell environment. We demonstrated the efficiency of this approach by the successful optimization of hit $\mathbf{2}$ into compound $\mathbf{3}$ endowed with an improved activity. In addition, compound $\mathbf{3}$ exhibits good antimicrobial activity against $S$. pneumoniae strains. In sum, our results show that it is possible to develop small-molecule inhibitors that block the entrance of vitamins into the bacteria through inhibition of ECF transporters and thereby the growth of pathogenic bacteria. Our bacterial uptake assay is amenable for screening of compound libraries and enables the optimization of inhibitors of ECF transporters. In addition, this assay can be extended to other members of the ECF transporter family by changing the natural substrate used for the uptake, providing a versatile screening platform.

\section{AUTHOR INFORMATION}

Corresponding Author

* Prof. A. K. H. Hirsch - Helmholtz Institute for Pharmaceutical Research Saarland (HIPS)Helmholtz Centre for Infection Research (HZI) Campus Building E8.1, 66123 Saarbrücken, Germany;

Department of Pharmacy, Saarland University, Campus Building E8.1, 66123 Saarbrücken, Germany; Stratingh Institute for Chemistry, University of Groningen, Nijenborgh 7, NL-9747 AG Groningen, the Netherlands. 
E-mail: anna.hirsch@helmholtz-hips.de

Author Contributions

The manuscript was written through contributions of all authors. All authors have given approval to the final version of the manuscript. $\ddagger$ These authors contributed equally. (match statement to author names with a symbol)

Funding Sources

A.K.H.H. gratefully acknowledges funding from the Netherlands Organization for Scientific Research (ChemThem and VIDI grants). A.K.H.H. acknowledges funding from the European Research Council (ERC starting grant 757913), the European Union's Horizon 2020 research and innovation programme under the Marie Skłodowska-Curie grant agreement No 713482 and the Helmholtz-Association's Initiative and Networking Fund. S.B. received ALERT grant (No 665250), the European Union's Horizon 2020 research and innovation programme under the Marie Skłodowska-Curie grant agreement No 713482.

Notes

Any additional relevant notes should be placed here.

\section{ACKNOWLEDGMENTS}

We thank Jeannine Jung for the cytotoxicity evaluation and Dr. Jennifer Herrmann, Prof. Dr. Rolf Müller for their guidance and insights. The authors would like to thank also Viktoria Schmitt and Stefanie Schmidt for antibacterial testing and Prof. Dr. Dirk Jan Slotboom and Dr. Inda Setyawati for their insights on the ECF transporters. 


\section{ABBREVIATIONS}

ECF, energy coupling factor; ABC, ATP-binding cassette; SAR, structure activity relationship;

OD, optical density; $K_{M}$, Michaelis constant; DMSO, dimethyl sulfoxide; Ar, aryl

\section{References}

(1) Rodionov, D. A.; Hebbeln, P.; Eudes, A.; ter Beek, J.; Rodionova, I. A.; Erkens, G. B.; Slotboom, D. J.; Gelfand, M. S.; Osterman, A. L.; Hanson, A. D., Eitinger, T. A novel class of modular transporters for vitamins in prokaryotes. J. Bacteriol. 2009, 191, 42-51.

(2) Rodionov, D. A.; Hebbeln, P.; Gelfand, M. S.; Eitinger, T. Comparative and functional genomic analysis of prokaryotic nickel and cobalt uptake transporters: evidence for a novel group of ATP-binding cassette transporters. J. Bacteriol.2006, 188, 317-327.

(3) Eudes, A.; Erkens, G. B.; Slotboom, D. J.; Rodionov, D. A.; Naponelli, V.; Hanson, A. D. Identification of genes encoding the folate- and thiamine-binding membrane proteins in Firmicutes. J. Bacteriol.2008, 190, 7591-7594.

(4) Bousis, S.; Setyawati, I.; Diamanti, E.; Slotboom, D. J.; Hirsch, A. K. H. Energy-Coupling Factor Transporters as Novel Antimicrobial Targets. Adv. Therap. 2018, 233, 1800066.

(5) Rice, L. B. Federal funding for the study of antimicrobial resistance in nosocomial pathogens: no ESKAPE. J.Infect. Dis.2008, 197, 1079-1081.

(6) World health organization. WHO priority pathogens list for R\&D of new antibiotics. http:// www.who.int/en/news-room/detail/27-02-2017-who-publishes-list-of-bacteria-for-which-newantibiotics-are-urgently-needed (accessed 04,2018).

(7) Henderson, G. B.; Zevely, E. M.; Huennekens, F. M. Mechanism of folate transport in Lactobacillus casei: evidence for a component shared with the thiamine and biotin transport systems. J. Bacteriol.1979, 137, 1308-1314.

(8) Diamanti E., Setyawati I., Bousis S., Souza P.C.T., Mojas L., Swier L., Haupenthal J., Gibson P., Volz C., Stanek W., Jaeger M., Herrmann J., Marrink S., Veening J-W., Müller R., Slotboom D.J., Hirsch A.K.H., 10.26434/chemrxiv-2021-xq08b-v2.

(9) Henderson, G. B.; Zevely, E. M.; Huennekens, F. M. Coupling of energy to folate transport in Lactobacillus casei. J. Bacteriol. 1979, 139, 552-559. 